\title{
COHOMOLOGY OF NILPOTENT SUBALGEBRAS OF AFFINE LIE ALGEBRAS
}

\author{
A. FIALOWSKI \\ (Communicated by Roe Goodman)
}

\begin{abstract}
We compute the cohomology of the maximal nilpotent Lie algebra of an affine Lie algebra $\hat{\mathfrak{g}}$ with coefficients in modules of functions on the circle with values in a representation space of $\mathfrak{g}$. These modules are not highest weight modules and are somewhat similar to the adjoint representation.
\end{abstract}

\section{INTRODUCTION}

Let $\mathfrak{g}$ be a finite-dimensional semisimple Lie algebra, $\mathfrak{b}$ a Borel subalgebra of $\mathfrak{g}$, and $\mathfrak{n}_{+} \subset \mathfrak{b}$ the maximal nilpotent ideal of $\mathfrak{b}$. The Bott-Kostant Theorem for Lie algebra cohomology is the following.

Theorem $[\mathrm{K}]$. Let $V$ be an irreducible representation of $\mathfrak{g}$ with dominant highest weight and $\mathfrak{n}$ a maximal nilpotent subalgebra of $\mathfrak{g}$. Then $\operatorname{dim} H^{i}(\mathfrak{n} ; V)$ is equal to the number of elements of length $i$ in the Weyl group of $\mathfrak{g}$.

Consider the affine infinite-dimensional graded Lie algebra $\hat{\mathfrak{g}}=\mathfrak{g} \otimes \mathbb{C}\left[t, t^{-1}\right]$ corresponding to $\mathfrak{g}$, with $\hat{\mathfrak{g}}_{i}=\mathfrak{g} \otimes t^{i}$. There are at least two analogues of the above Theorem for affine algebras. The most direct analogue is the following: if $V$ is an irreducible representation of the current algebra $\hat{\mathfrak{g}}$ with dominant highest weight and $\hat{\mathfrak{n}}_{+}$is a maximal nilpotent subalgebra of $\hat{\mathfrak{g}}$, that is,

$$
\hat{\mathfrak{n}}_{+}=\left(\mathfrak{n}_{+} \otimes 1\right) \oplus(\mathfrak{g} \otimes t) \oplus\left(\mathfrak{g} \otimes t^{2}\right) \oplus \cdots,
$$

then $\operatorname{dim} H^{i}\left(\hat{\mathfrak{n}}_{+} ; V\right)$ is equal to the number of elements of length $i$ in the Weyl group. This Theorem was proved by Garland in 1975 [G] and Garland and Lepowsky in 1976 (see [GL]). The proof is similar to that of the finitedimensional case.

In this paper we present the proof of a different analogue of the Bott- Kostant Theorem obtained jointly with Feigin and announced in [FF]. Namely, we compute the cohomology of $\hat{\mathfrak{n}}_{+}$with coefficients in modules of functions on the

Received by the editors May 11, 1992 and, in revised form, December 15, 1992.

1991 Mathematics Subject Classification. Primary 17B56, 18B67, 17B30; Secondary 22E41, 22E60.

Key words and phrases. Affine Lie algebra, adjoint representation, Lie algebra cohomology, spectral sequence. 
circle $S^{1}$ with values in a representation space of $\mathfrak{g}$. These modules are not highest weight modules and are somewhat similar to the adjoint representation.

\section{RESULTS}

Let $V$ be a representation of $\mathfrak{g}, A$ a $\mathbb{C}$-algebra, and $\varphi: \mathbb{C}\left[t, t^{-1}\right] \rightarrow A$ a homomorphism. Let us define a representation of $\hat{\mathfrak{g}}$ on $V \otimes A$ by

$$
(x \otimes f)(v \otimes a)=x(v) \otimes \varphi(f) a,
$$

where $x \in \mathfrak{g}, v \in V, f \in \mathbb{C}\left[t, t^{-1}\right]$, and $a \in A$.

Consider two special cases for $A$ and $\varphi$ :

(a) $A=\mathbb{C}\left[t, t^{-1}\right]$ and $\varphi=$ id. In this case denote the module $V \otimes A$ by $\hat{V}$. It consists of rational functions $\mathbb{C} \rightarrow V$ that are regular outside the origin.

(b) $A=\mathbb{C}$ and $\varphi(f)=f(1)$. In this case denote the module $V \otimes A$ by $V_{1}$.

Note that the map assigning to a function $\mathbb{C} \rightarrow V$ its value at 1 defines a homomorphism $\hat{V} \rightarrow V_{1}$. The space $\hat{V}$ is endowed with an obvious module structure over the algebra $\mathbb{C}\left[t, t^{-1}\right]$, and it is easy to see that multiplication by an element of $\mathbb{C}\left[t, t^{-1}\right]$ is a $\mathfrak{g}$-endomorphism of the $\hat{\mathfrak{g}}$-module $\hat{V}$. Finally, note that $\hat{V}$ is a graded $\hat{\mathfrak{g}}$-module, that is, $\hat{V}=\bigoplus_{i \in \mathbf{Z}} \hat{V}_{i}$, with $\hat{V}_{i}=V \otimes t^{i}$.

First we will compute the cohomology of $\hat{n}_{+}$with coefficients in $\hat{V}$. The Lie algebra $\hat{\mathfrak{n}}_{+}$is a graded subalgebra of $\hat{\mathfrak{g}}$, and $\hat{V}$ is a graded $\hat{\mathfrak{g}}$-module and $\hat{\mathfrak{n}}_{+}-$ module. Denote by $C^{*}\left(\hat{\mathfrak{n}}_{+} ; \hat{V}\right)$ the cochain complex of $\hat{\mathfrak{n}}_{+}$with coefficients in the $\hat{\mathfrak{n}}_{+}$-module $\hat{V}$. The complex $C^{*}\left(\hat{\mathfrak{n}}_{+} ; \hat{V}\right)$ and the cohomology $H^{*}\left(\hat{\mathfrak{n}}_{+} ; \hat{V}\right)$ are graded by weights. To state this, we introduce the notation

$$
C_{(m)}^{*}\left(\hat{\mathfrak{n}}_{+} ; \hat{V}\right)=\bigoplus_{r \in \mathbb{Z}} \operatorname{Hom}\left(\left(\bigwedge^{q} \hat{\mathfrak{n}}_{+}\right)_{r}, \hat{V}_{r+m}\right),
$$

where

$$
\left(\bigwedge^{q} \hat{\mathfrak{n}}_{+}\right)_{r}=\Lambda^{q} \hat{\mathfrak{n}}_{+} \cap\left(\bigotimes^{q} \hat{\mathfrak{n}}_{+}\right)_{r}
$$

and

$$
\left(\bigotimes^{q} \hat{\mathfrak{n}}_{+}\right)_{r}=\bigoplus_{r_{1}+\cdots+r_{q}=r}\left(\left(\hat{\mathfrak{n}}_{+}\right)_{r_{1}} \otimes \cdots \otimes\left(\hat{\mathfrak{n}}_{+}\right)_{r_{q}}\right) .
$$

In this notation the grading is

$$
C^{*}\left(\hat{\mathfrak{n}}_{+} ; \hat{V}\right)=\bigoplus_{m \in \mathbf{Z}} C_{(m)}^{*}\left(\hat{\mathfrak{n}}_{+} ; \hat{V}\right)
$$

and, similarly,

$$
H^{*}\left(\hat{\mathfrak{n}}_{+} ; \hat{V}\right)=\bigoplus_{m \in \mathbf{Z}} H_{(m)}^{*}\left(\hat{\mathfrak{n}}_{+} ; \hat{V}\right) .
$$

Lemma 1. $H_{(m)}^{*}\left(\hat{\mathfrak{n}}_{+} ; \hat{V}\right) \cong H^{*}\left(\hat{\mathfrak{n}}_{+} ; V_{1}\right)$ for all $m \in \mathbb{Z}$.

Proof. The composition of mappings

$$
C_{(m)}^{*}\left(\hat{\mathfrak{n}}_{+} ; \hat{V}\right) \stackrel{i}{\longrightarrow} C^{*}\left(\hat{\mathfrak{n}}_{+} ; \hat{V}\right) \stackrel{s}{\longrightarrow} C^{*}\left(\hat{\mathfrak{n}}_{+} ; V_{1}\right),
$$


where $i$ is the embedding and $s$ is induced by the homomorphism $\hat{V} \rightarrow V_{1}$, is obviously a complex isomorphism.

Moreover, the isomorphisms

$$
\hat{V}_{i}=V \otimes t^{i} \longrightarrow V \otimes t^{i+1}=\hat{V}_{i+1}
$$

define a $\hat{\mathfrak{g}}$-isomorphism $t: \hat{V} \rightarrow \hat{V}$ of degree 1 , which generates an action of $\mathbb{C}\left[t, t^{-1}\right]$ in $\hat{V}$ and in $H^{*}\left(\hat{\mathfrak{n}}_{+} ; \hat{V}\right)$. Evidently, $t$ maps $H_{(m)}^{*}\left(\hat{\mathfrak{n}}_{+} ; \hat{V}\right)$ isomorphically onto $H_{(m+1)}^{*}\left(\hat{n}_{+} ; \hat{V}\right)$. Hence we have

Lemma $1^{\prime} \cdot H^{*}\left(\hat{\mathfrak{n}}_{+} ; \hat{V}\right) \cong \mathbb{C}\left[t, t^{-1}\right] \otimes_{\mathbb{C}} H^{*}\left(\hat{\mathfrak{n}}_{+} ; V_{1}\right)$.

Now let us compute the cohomology $H^{*}\left(\hat{\mathfrak{n}}_{+} ; V_{1}\right)$. Introduce the subalgebra $\mathfrak{g}[t]=\mathfrak{g} \otimes \mathbb{C}[t]$ of $\hat{\mathfrak{g}}$. In the following we will identify $\mathfrak{g}$ with $\mathfrak{g} \otimes 1 \subset \mathfrak{g}[t]$. The Lie algebra $\hat{\mathfrak{n}}_{+}$is embedded into $\mathfrak{g}[t]$, and $V_{1}$ is naturally endowed with a $\mathfrak{g}[t]$-module structure.

Theorem 1. We have the following isomorphism of cohomology spaces:

$$
H^{i}\left(\hat{\mathfrak{n}}_{+} ; V_{1}\right) \stackrel{\cong}{\longrightarrow} \bigoplus_{p+q=i} H^{p}\left(\hat{\mathfrak{n}}_{+} ; \mathbb{C}\right) \otimes H^{q}\left(\mathfrak{g}[t], \mathfrak{g} ; V_{1}\right)
$$

Proof. We begin the proof, which will take most of this paper, by introducing two subalgebras of $\hat{\mathfrak{g}}$. The first is

$$
\overline{\mathfrak{g}}=(t-1) \mathfrak{g} \oplus(t-1)^{2} \mathfrak{g} \oplus \cdots
$$

consisting of loops $\varphi(t)$ which vanish at 1 , and the second is

$$
\overline{\mathfrak{n}}=\hat{\mathfrak{n}}_{+} \cap \overline{\mathfrak{g}} \text {. }
$$

Note that $\mathfrak{g}[t]=\hat{\mathfrak{n}}_{+}+\overline{\mathfrak{g}}$. Let $G$ be the compact connected, simply connected Lie group, corresponding to the compact real form of $\mathfrak{g}$. Next we need the following theorem.

Theorem 2. We have

$$
H^{*}(\overline{\mathfrak{n}}) \cong H^{*}\left(\hat{\mathfrak{n}}_{+}\right) \otimes H^{*}(\overline{\mathfrak{g}}) \otimes H^{*}(\Omega G),
$$

where $\Omega G$ is the loop space of $G$.

Proof. Since we have the embeddings

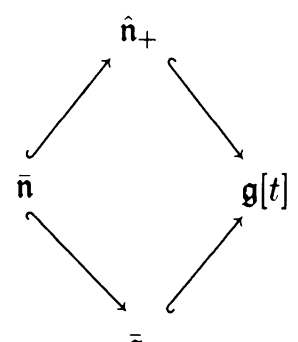


with $\overline{\mathfrak{g}} \mapsto \mathfrak{g}[t] \mapsto \mathfrak{g}$, we also have the diagram:

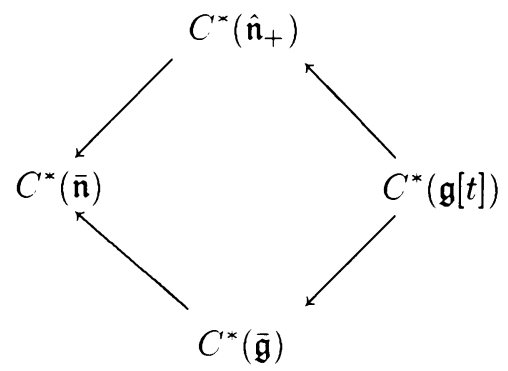

Consequently,

$$
C^{*}(\overline{\mathfrak{n}})=C^{*}\left(\hat{\mathfrak{n}}_{+}\right) \otimes_{C^{*}(\mathfrak{g}[t])} C^{*}(\overline{\mathfrak{g}})
$$

where the tensor product is taken in the category of differential algebras. In such a situation there exists an Eilenberg-Moore spectral sequence, connecting these four differential algebras. Its second term is

$$
E_{2}=\operatorname{Tor}_{H^{*}(\mathfrak{g}[t])}\left(H^{*}\left(\hat{\mathfrak{n}}_{+}\right), H^{*}(\overline{\mathfrak{g}})\right),
$$

and its limit term is $H^{*}(\overline{\mathfrak{n}})$. We know that $H^{*}(\mathfrak{g}[t]) \cong H^{*}(\mathfrak{g})$ (see, for example, [F]). On the other hand, since $H^{*}(\mathfrak{g})$ acts trivially on $H^{*}(\overline{\mathfrak{g}})$ and also on $H^{*}\left(\hat{\mathfrak{n}}_{+}\right)$, we conclude that the composition $H^{*}(\mathfrak{g}) \rightarrow H^{*}\left(\mathfrak{n}_{+}\right) \rightarrow H^{*}\left(\hat{\mathfrak{n}}_{+}\right)$is trivial. So we have

$$
E_{2}=\operatorname{Tor}_{H^{*}(\mathfrak{g})}\left(H^{*}\left(\hat{\mathfrak{n}}_{+}\right), H^{*}(\overline{\mathfrak{g}})\right) \cong H^{*}\left(\hat{\mathfrak{n}}_{+}\right) \otimes H^{*}(\overline{\mathfrak{g}}) \otimes \operatorname{Tor}_{H^{*}(\mathfrak{g})}(\mathbb{C}, \mathbb{C}) .
$$

On the other hand,

$$
\operatorname{Tor}_{H^{*}(\mathfrak{g})}(\mathbb{C}, \mathbb{C}) \cong H^{*}(\Omega G) \text {. }
$$

Indeed, the cohomology algebra of $\mathfrak{g}$ with trivial coefficients coincides with the cohomology algebra of $G$, and by the Hopf Theorem it is commutative and free (see [S]). Using the computation of $\operatorname{Tor}_{A}(\mathbb{C}, \mathbb{C})$ for the free commutative algebra $A$ ([M, Proposition 7.3] and see also [A]) and the connection between the cohomology of $G$ and $\Omega G$, we get the isomorphism $\operatorname{Tor}_{A}(\mathbb{C}, \mathbb{C}) \cong H^{*}(\Omega G)$ :

$$
H^{*}(\mathfrak{g})=\bigwedge^{*}\left(e_{\alpha_{1}}, \ldots, e_{\alpha_{k}}\right),
$$

where $e_{\alpha_{i}} \in H^{\alpha_{i}}$. So with the mapping $G \rightarrow \Omega G$ we have

$$
\operatorname{Tor} \bigwedge^{*}\left(e_{\alpha_{1}}, \ldots, e_{\alpha_{k}}\right)(\mathbb{C}, \mathbb{C})=S^{*}\left(c_{\alpha_{1}-1}, \ldots, c_{\alpha_{k}-1}\right),
$$

where $\operatorname{deg} c_{\alpha_{i}-1}=\alpha_{i}-1$. The generators of the cohomology are the homotopy groups. To complete the proof of Theorem 2 we will need the next proposition.

Proposition 1. The spectral sequence degenerates, namely, its second term $E_{2}$ coincides with the limit term $E_{\infty}$.

Proof. We shall indicate explicit cocycles of $C^{*}(\overline{\mathfrak{n}})$ which represent the generators of $E_{2}$. For this we apply the continuous cohomology theory. Let $\mathfrak{n}(0,1)$ be the Lie algebra of infinitely differentiable functions $f:[0,1] \rightarrow \mathfrak{g}$ such that $f(0) \in \mathfrak{n}$ and $f(1)=0$. Denote by $C_{c}^{*}(0,1)$ the complex of cochains of $\mathfrak{n}(0,1)$, continuous in the $C^{\infty}$-topology. Let $\alpha$ be a generator of $H^{*}(\mathfrak{g})$ and 
$\bar{\alpha}$ a cochain representing $\alpha$. For $p \in[0,1]$ denote by $\varphi_{p}$ the homomorphism $\overline{\mathfrak{n}} \rightarrow \mathfrak{g}$, "the value at $p$ ":

$$
\varphi_{p}\left((t-1) g_{1},(t-1)^{2} g_{2}, \ldots\right)=\sum_{m}(p-1)^{m} g_{m} .
$$

Let $\alpha_{p}=\varphi_{p}^{*} \bar{\alpha}, \alpha_{p} \in C_{c}^{*}(0,1)$. Choose $\bar{\alpha}$ in such a way that $\alpha_{0}=\alpha_{1}=0$. Let $p \neq 0,1$; then we can define the cochain $\frac{\partial \alpha}{\partial x}(p)$, where $x$ is the coordinate on $[0,1]$. It is shown in [F] that $\frac{\partial \alpha}{\partial x}(p)$ is a coboundary: $\frac{\partial \alpha}{\partial x}(p)=\delta \omega(p)$, where $\delta$ is the differential in $C_{c}^{*}(0,1)$. Indeed, let $K_{p}(p \neq 0)$ be the cochain complex of $\overline{\mathfrak{n}}$ with support at $p$. It is proved in the same paper that the cohomology of $K_{p}$ is isomorphic to $H^{*}(\mathfrak{g})$. The complex $K_{p}$ is a $W_{1}$-module, where $W_{1}$ is the Lie algebra of formal vector fields at the point $p$. But $H^{*}(\mathfrak{g})$ is finite dimensional and $W_{1}$ has no nontrivial finite-dimensional representations. We conclude that if $\nu \in K_{p}$ and $\delta \nu=0$ then $\frac{\partial \nu}{\partial x}$ is the differential of some other cocycle $\bar{\nu} \in K_{p}$.

This means that

$$
\alpha_{p}-\alpha_{q}=\delta \int_{q}^{p} \omega(x) d x .
$$

In particular, $\delta \int_{0}^{1} \omega(x) d x=0$. Suppose that $\alpha^{\prime}=\int_{0}^{1} \omega(x) d x$. The cochain $\alpha^{\prime}$ represents a nontrivial cohomology class of $\overline{\mathfrak{n}}$.

The Lie algebras $\hat{\mathfrak{n}}_{+}$and $\overline{\mathfrak{g}}=(t-1) \mathfrak{g} \oplus(t-1)^{2} \mathfrak{g} \oplus \cdots$ are graded. Similarly, the cochain complexes are also graded. Note that the cochain complex $K_{0}$ of $\mathfrak{n}(0,1)$ with support in 0 is isomorphic to $\bigoplus_{i} C_{i}^{*}\left(\hat{\mathfrak{n}}_{+}\right)$and the cochain complex $K_{1}$ with support in 1 is isomorphic to $\bigoplus_{i} C_{i}^{*}(\overline{\mathfrak{g}})$. It follows from this that the cohomologies of $K_{0}$ and $K_{1}$ are isomorphic to $H^{*}\left(\hat{\mathfrak{n}}_{+}\right)$and $H^{*}(\overline{\mathfrak{g}})$, respectively.

Recall that $H^{*}(\mathfrak{g})$ is isomorphic to the free graded commutative algebra with generators $\xi_{1}, \xi_{2}, \ldots$, with $\operatorname{deg} \xi_{k}=2 k+1$. Using the above construction, let us assign to each $\xi_{i}$ a representative cocycle $\xi_{i}^{\prime}$.

Lemma 2. The space $H^{*}(\overline{\mathfrak{n}})$ is generated by the cohomology classes of cochains of the form $u \wedge v \wedge P\left(\xi_{1}^{\prime}, \xi_{2}^{\prime}, \ldots\right)$, where $u \in K_{0}$ and $v \in K_{1}$ are cocycles corresponding to the elements of $H^{*}\left(\hat{\mathfrak{n}}_{+}\right)$and $H^{*}(\overline{\mathfrak{g}})$, respectively, and $P$ is an arbitrary polynomial with generators $\xi_{1}^{\prime}, \xi_{2}^{\prime}, \ldots$.

The proof of Lemma 2 follows from the construction above for continuous cohomology (a similar argument in a more difficult situation was used in [FR]). In particular, we have an explicit construction of cochains, representing the generators of $E_{2}$ in the proof of Theorem 2, surviving until $E_{\infty}$. Thus, Theorem 2 is proved.

We now return to complete the proof of Theorem 1 . We want to prove the isomorphism

$$
H^{*}\left(\mathfrak{g}[t], \mathfrak{g} ; V_{1}\right) \otimes H^{*}\left(\hat{\mathfrak{n}}_{+} ; \mathbb{C}\right) \stackrel{\sim}{\longrightarrow} H^{*}\left(\hat{\mathfrak{n}}_{+} ; V_{1}\right) .
$$

Consider the Serre-Hochschild spectral sequence associated with the algebra $\hat{\mathfrak{n}}_{+}$, its ideal $\overline{\mathfrak{n}}$, and the module $V_{1}$. The Lie algebra $\overline{\mathfrak{n}}$ acts on $V_{1}$ trivially. The second term of this spectral sequence is

$$
E_{2}^{i j}=H^{i}\left(\hat{\mathfrak{n}}_{+} / \overline{\mathfrak{n}} ; H^{j}\left(\overline{\mathfrak{n}} ; V_{1}\right)\right)=H^{i}\left(\hat{\mathfrak{n}}_{+} / \overline{\mathfrak{n}} ; H^{j}(\overline{\mathfrak{n}} ; \mathbb{C}) \otimes V_{1}\right) .
$$


But by Theorem 2, this is the same as

$$
H^{i}\left(\hat{\mathfrak{n}}_{+} / \overline{\mathfrak{n}}, \bigoplus_{p+q+r=j} H^{p}\left(\hat{\mathfrak{n}}_{+}\right) \otimes H^{q}(\overline{\mathfrak{g}}) \otimes H^{r}(\Omega G) \otimes V_{1}\right) .
$$

Since $\hat{\mathfrak{n}}_{+} / \overline{\mathfrak{n}} \cong \mathfrak{g}$, we get that $E_{2}$ is then isomorphic to

$$
H^{*}(\mathfrak{g}, \mathbb{C}) \otimes\left[H^{*}\left(\hat{\mathfrak{n}}_{+}\right) \otimes H^{*}(\overline{\mathfrak{g}}) \otimes H^{*}(\Omega G) \otimes V_{1}\right]^{\mathfrak{g}},
$$

where [ $]^{\mathfrak{g}}$ denotes the invariant space.

Let us note the following facts:

(a) $\mathfrak{g}$ acts on $H^{*}\left(\hat{\mathfrak{n}}_{+}\right)$trivially (this action is extended by the projection $\hat{\mathfrak{n}}_{+} \rightarrow \mathfrak{g}$ to the canonical action of $\hat{\mathfrak{n}}_{+}$, and a Lie algebra acts trivially on the cohomology of itself).

(b) $\mathfrak{g}$ acts trivially on $H^{*}(\Omega G)=\operatorname{Tor}_{H^{*}(\mathfrak{g})}(\mathbb{C}, \mathbb{C})$.

These imply that

$$
E_{2}^{i j}=H^{i}(\mathfrak{g}) \otimes\left(\bigoplus_{p+q+r=j}\left(H^{p}\left(\hat{\mathfrak{n}}_{+}\right) \otimes H^{q}(\Omega G) \otimes\left[H^{r}(\overline{\mathfrak{g}}) \otimes V_{1}\right]^{\mathfrak{g}}\right)\right)
$$

and

$$
\left[H^{*}(\overline{\mathfrak{g}}) \otimes V_{1}\right]^{\mathfrak{g}}=H^{*}\left(\mathfrak{g}[t], \mathfrak{g} ; V_{1}\right) .
$$

The differentials act in the following way.

(i) Differentials on $H^{*}\left(\hat{\mathfrak{n}}_{+}\right) \otimes H^{*}\left(\mathfrak{g}[t], \mathfrak{g} ; V_{1}\right)$ are zero. We have the map

$$
H^{*}\left(\hat{\mathfrak{n}}_{+}\right) \otimes H^{*}\left(\mathfrak{g}[t], \mathfrak{g} ; V_{1}\right) \longrightarrow H^{*}\left(\hat{\mathfrak{n}}_{+} ; V_{1}\right) \text {. }
$$

Thus, elements of the left side survive in $E_{\infty}$.

(ii) Differentials on

$$
H^{*}(\mathfrak{g}) \otimes H^{*}(\Omega G)=\bigwedge^{*}\left\{e_{\alpha_{i}}\right\} \otimes S^{*}\left\{c_{\alpha_{i}-1}\right\}
$$

map the generators of the algebra $H^{*}(\Omega G)$ into the generators of $H^{*}(g)$ (the differential maps $\left.c_{\alpha_{i}-1} \mapsto e_{\alpha_{i}}\right)$.

Consider the Serre path fibration $E G \rightarrow G$. Since the paths are contractible, $H^{0}(E G)=\mathbb{C}$ and $H^{i}(E G)=0$ for $i>0$. In addition, we have $H^{*}(\mathfrak{g})=$ $H^{*}(G)$, so

$$
H^{*}(G) \otimes H^{*}(\Omega G) \text { converges to } H^{*}(E G) \cong \mathbb{C} .
$$

Then it follows that the spectral sequence converges to $H^{*}\left(\hat{\mathfrak{n}}_{+}\right) \otimes H^{*}\left(\mathfrak{g}[t], \mathfrak{g} ; V_{1}\right)$. Thus our spectral sequence is the product of $H^{*}\left(\hat{\mathfrak{n}}_{+}\right) \otimes H^{*}\left(\mathfrak{g}[t], \mathfrak{g} ; V_{1}\right)$ with the spectral sequence of the Serre path fibration $E G \rightarrow G$. This completes the proof of Theorem 1.

Theorem 3. $H^{i}\left(\hat{\mathfrak{n}}_{+} ; \hat{\mathfrak{g}}\right) \cong \mathbb{C}\left[t, t^{-1}\right] \otimes_{\mathbb{C}} H^{i-1}\left(\hat{\mathfrak{n}}_{+}\right)$for any nonnegative integer $i$. Proof. Let $V=\mathfrak{g}$ as in Lemma $1^{\prime}$. Then Lemma $1^{\prime}$ implies that $H^{i}\left(\hat{\mathfrak{n}}_{+} ; \hat{\mathfrak{g}}\right)$ is a free $\mathbb{C}\left[t, t^{-1}\right]$-module of rank equal to $\operatorname{dim} H^{i}\left(\hat{\mathbf{n}}_{+} ; V_{1}\right)$.

The cohomology of $\hat{\mathfrak{n}}_{+}$with trivial coefficients is known (see [GL]). Using this result, it is not difficult to find the cohomology $H^{*}(\operatorname{tg}[t])$ of the algebra $t \mathfrak{g}[t]=(\mathfrak{g} \otimes t) \oplus\left(\mathfrak{g} \otimes t^{2}\right) \oplus \cdots$ (see also [G] and [GL]; the proof in [GL] is basically the same as the proof of the cohomology result of $\hat{\mathfrak{n}}_{+}$with trivial coefficients). We need only the following fact. The space $H^{*}(t \mathfrak{g}[t])$ is a $\mathfrak{g}$-module 
and $\operatorname{Hom}_{\mathfrak{g}}\left(\mathfrak{g} ; H^{i}(t \mathfrak{g}[t])\right)=0$ if $i \neq 1$ and is equal to $\mathbb{C}$ if $i=1$ (see [L]). Since $H^{i}(\mathfrak{g}[t], \mathfrak{g} ; V) \cong \operatorname{Hom}_{\mathfrak{g}}\left(V ; H^{i}(t \mathfrak{g}[t])\right)$, this gives us that $H^{i}(\mathfrak{g}[t], \mathfrak{g} ; \mathfrak{g})=0$ for $i \neq 1$ and is equal to $\mathbb{C}$ for $i=1$. After this, it is enough to apply Theorem 1 to find that $H^{i}\left(\hat{\mathfrak{n}}_{+} ; V_{1}\right)=H^{i-1}\left(\hat{\mathfrak{n}}_{+}\right)$.

\section{ACKNOWLEDGMENT}

The authors would like to thank the referee for the useful comments.

\section{REFERENCES}

[A] L. L. Avramov, On the Hopf algebra of a local ring, Izv. Akad. Nauk SSSR Ser. Mat. 38 (1974), 253-277.

[F] B. L. Feigin, Cohomology of groups and algebras of flows, Russian Math. Surveys 35 (1980), 239-240.

[FF] B. L. Feigin and A. Fialowski, The cohomology of nilpotent algebras of flows, Soviet Math. Dokl. 28 (1983), no. 1, 178-181.

[FR] B. L. Feigin. and V. S. Retach, Cohomology of some Lie algebras and superalgebras of vector fields, Russian Math. Surveys 37 (1982), no. 2, 251-252.

[Fu] D. B. Fuchs, Cohomology of infinite dimensional Lie algebras, Translation from Russian, Consultants Bureau, New York, 1986.

[G] H. Garland, Dedekind's $\zeta$-function and the cohomology of infinite dimensional Lie algebras, Proc. Nat. Acad. Sci. U.S.A. 72 (1975), 2493-2495.

[GL] H. Garland and J. Lepowsky, Lie algebra homology and the Macdonald-Kac formulas, Invent. Math. 34 (1976), 37-76.

[K] B. Kostant, Lie algebra cohomology and the generalized Borel-Weil Theorem, Ann. of Math. (2) 74 (1961), 329-387.

[L] J. Lepowsky, Generalized Verma modules, loop space cohomology and Macdonald-type identities, Ann. Sci. École Norm. Sup. (4) 12 (1979), 169-235.

[M] J. C. Moore, Algebre homologique et homologie espace classificants, Sem. H. Cartan, Expose 7, 1959/60.

[S] J.-P. Serre, Groupes d'homotopie et classes de groupes abeliens, Ann. of Math. (2) 58 (1953), 258-294.

Department of Mathematics, University of California, Davis, California 95616-8633

E-mail address: fialowsk@math.ucdavis.edu 